\title{
Improved LMI-based stability conditions for a competitive Lotka-Volterra system with time-varying delays
}

\author{
Rui Dong ${ }^{1}$, Lei Chen ${ }^{1}$ and Yonggang Chen ${ }^{1 *}$
}

"Correspondence:

happycygzmd@tom.com 'School of Mathematical Sciences, Henan Institute of Science and Technology, Xinxiang, China

\section{Springer}

\begin{abstract}
This paper is concerned with the local asymptotic stability problem for a competitive Lotka-Volterra system with time-varying delays. By constructing the novel augmented Lyapunov-Krasovskii functionals and using the Wirtinger integral inequality, some alternative stability criteria are obtained by means of linear matrix inequalities. In particular, our constructed functionals contain some cross terms related to four different time delays. The proposed stability conditions in this paper are less conservative as compared with the most existing ones due to using some advanced techniques. Finally, a numerical example illustrates the effectiveness and improvement of the obtained results.
\end{abstract}

Keywords: Asymptotic stability; Lotka-Volterra system; Time-varying delays; Linear matrix inequalities

\section{Introduction}

More than sixty years ago, Lotka and Volterra laid the foundation for the science of competition between different species in $[1,2]$. Over the several decades, considerable work has been done for $\mathrm{N}$-species Lotka-Volterra (L-V) type systems to discuss their persistence, extinction, and global or local asymptotic behavior in [3-16]. For example,the interspecific competition has been observed based on the experiments in [4], partial persistence and extinction in $\mathrm{N}$-dimensional competitive systems have been addressed in [6], and [7] shows a discrete delay decomposition approach to analyse stability of linear retarded and neutral systems. The permanence of stochastic L-V systems has been analyzed in [8-12]. In [13], sufficient and necessary conditions for permanence and extinction in a three-dimensional competitive L-V system have been proposed. In [14], a two species non-autonomous competitive phytoplankton system with Beddington-DeAngelis functional response has been proposed and sufficient conditions that guarantee the extinction of a species and global attractivity of the other one have been obtained. The stability analysis problem for competitive L-V system has also been one of the interesting research issues, and its global and local asymptotic stability problems have been sufficiently focused in [12, 14-16].

Considering the important influence of time delays on the system, there is considerable work on the persistence, extinction, and asymptotical stability of L-V competitive systems with delays in [3, 17-24]. For example, in [3] Teng has established the criteria un-

(c) The Author(s) 2019. This article is distributed under the terms of the Creative Commons Attribution 4.0 International License (http://creativecommons.org/licenses/by/4.0/), which permits unrestricted use, distribution, and reproduction in any medium, provided you give appropriate credit to the original author(s) and the source, provide a link to the Creative Commons license, and indicate if changes were made. 
der which a part of species is driven to extinction while other are persistent or all species are coexistent globally asymptotically stable. In [17], Oca and Pérez have extended the principle of competitive exclusion $\mathrm{N}$-species nonautonomous L-V competition systems of differential equations with infinite delay, and in [18], Qiu and Deng have studied the optimal harvesting of a stochastic delay competitive L-V model with Lévy jumps systematically. For the stability analysis of time-delay systems, it has been identified that the Lyapunov-Krasovskii (L-K) theory based on the L-K functionals is less conservative than the Lyapunov-Razumikhin approach. By constructing some appropriate L-K functionals, the conditions for global or local asymptotic stability for competitive L-V systems with delays have been obtained in [3, 19-24]. By employing a linear matrix inequality (LMI) approach, a series of sufficient criteria which can ensure the stability of the positive equilibrium for the given L-V systems have been proposed in [22-24]. Specially, by using the LMI optimization approach, Park has obtained a stability criterion for the local stability of the positive equilibrium of the two-species L-V systems with discrete delays in [22]; Qiu and Cao have obtained sufficient criteria which guarantee the exponential stability of the same system in [23]; Sun and Meng have addressed the local asymptotic stability for the $\mathrm{L}-\mathrm{V}$ system with time-varying delays in [24].

The integral terms are common in the derivative of L-K functionals, and the overapproximation methods are used to replace the integral terms with some more effective expressions. Unavoidably, the use of any inequalities will introduce some conservatism in the analysis and consequently in the resulting stability conditions. Over the past two decades, the inequality $2 a b \leq a^{2}+b^{2}$ has been used in [21], the inequality $\sigma \int_{0}^{\sigma} w(s) d s \geq$ $\left[\int_{0}^{\sigma} w(s) d s\right]^{2}$ has been adopted in [22] and [23], and Jensen's inequality $[z(t)-z(t-$ $\left.\left.\tau_{i j}(t)\right)\right]^{T} W\left[z(t)-z\left(t-\tau_{i j}(t)\right)\right] \leq h_{i j} \int_{t-\tau_{i j}(t)}^{t} \dot{z}^{T}(s) W \dot{z}(s) d s$ has been employed in [24], respectively. The inherent conservative nature of these inequalities has led to some possible conservatism in the obtained conditions. Recently, the less conservative Wirtinger-based inequality has been proposed in $[25,26]$. By incorporating augmented L-K functionals, some more effective stability conditions have been established for linear time-delay systems in $[25,26]$. Unfortunately, for the competitive L-V system with time delays, the existing stability conditions are all based on the conservative inequalities and the simple L-K functionals. More importantly, the relationships between multiple delays are neglected in constructing the L-K functionals. Therefore, the existing results concerning the delayed L-V system are conservative to some extent, which motivates the present research.

In this paper, the following two-species competitive L-V system with time-varying delays is considered:

$$
\left\{\begin{array}{l}
\frac{d x(t)}{d t}=x(t)\left[b_{1}-a_{11} x\left(t-\tau_{11}(t)\right)-a_{12} y\left(t-\tau_{12}(t)\right)\right], \\
\frac{d y(t)}{d t}=y(t)\left[b_{2}-a_{21} x\left(t-\tau_{21}(t)\right)-a_{22} y\left(t-\tau_{22}(t)\right)\right],
\end{array}\right.
$$

where $x(t)$ and $y(t)$ stand for densities of both the populations at time $t$, respectively; $b_{i}, a_{i j}$ are positive scalars and $0 \leq \tau_{i j}(t) \leq \tau_{i j}, \dot{\tau}_{i j}(t) \leq d_{i j}$ with $d_{i j} \geq 0, \tau_{i j}>0(i, j=1,2)$. The initial condition of system (1) is given as

$$
\left\{\begin{array}{l}
x(s)=\phi_{1}(s) \geq 0, \quad-\tau \leq s \leq 0, \phi_{1}(0)>0, \\
y(s)=\phi_{2}(s) \geq 0, \quad-\tau \leq s \leq 0, \phi_{2}(0)>0
\end{array}\right.
$$

where $\tau=\max _{1 \leq i, j \leq 2}\left\{\tau_{i j}(t)\right\}$, and $\phi_{1}(s), \phi_{2}(s)$ are assumed to be continuous. 
If $a_{11} / a_{21}>b_{1} / b_{2}>a_{12} / a_{22}$, by denoting $z^{*}=\left(x^{*}, y^{*}\right)$ with $x^{*}=\frac{b_{1} a_{22}-b_{2} a_{12}}{a_{11} a_{22}-a_{12} a_{21}}, y^{*}=$ $\frac{b_{2} a_{11}-b_{1} a_{21}}{a_{11} a_{22}-a_{12} a_{21}}$, it is seen that $x^{*}, y^{*} \in(0,1)$. The four equilibrium points of system $(1)$ are $(0,0),\left(0, \frac{b_{2}}{a_{22}}\right),\left(\frac{b_{1}}{a_{11}}, 0\right)$, and $z^{*}$ respectively. Define all positive solutions $z(t)=(x(t), y(t))$ of system (1). The point $z^{*}$ is the only one positive equilibrium point. The equilibrium point $z^{*}$ indicates the coexistence of the two species.

In this paper, we are mainly concerned with the local stability problem of the positive equilibrium $z^{*}$ of system (1). To reduce the possible conservatism in analyzing the stability of time-delay systems, this paper will choose the novel augmented L-K functionals and the less conservative integral inequalities in [26]. In particular, it should be pointed out that the second L-K functional proposed in this paper includes the cross terms between different time delays. The developed stability conditions in this paper are less conservative as compared with the existing ones in [22-24]. A numerical example shows the effectiveness and benefit of the proposed results.

Notation. The superscript " $T$ " stands for the transpose of a matrix. The matrix $R>0$ means that $R$ is symmetric and positive definite, $\mathbb{R}^{n}$ denotes the $n$-dimensional Euclidean space. In the paper, $I$ and 0 are used to denote the identity and zero matrices with proper dimension. The symmetric terms in a symmetric matrix are denoted by $*$. In addition, we denote $\mathbf{H e}(A)=A+A^{T}$.

\section{Preliminaries}

Letting $u(t)=x(t)-x^{*}, v(t)=y(t)-y^{*}$, system (1) can be written as

$$
\left\{\begin{array}{l}
\dot{u}(t)=\left[u(t)+x^{*}\right]\left[-a_{11} u\left(t-\tau_{11}(t)\right)-a_{12} v\left(t-\tau_{12}(t)\right)\right], \\
\dot{v}(t)=\left[v(t)+y^{*}\right]\left[-a_{21} u\left(t-\tau_{21}(t)\right)-a_{22} v\left(t-\tau_{22}(t)\right)\right],
\end{array}\right.
$$

Correspondingly, the linearized model of (3) can be described by

$$
\left\{\begin{array}{l}
\dot{u}(t)=-a_{11} x^{*} u\left(t-\tau_{11}(t)\right)-a_{12} x^{*} v\left(t-\tau_{12}(t)\right), \\
\dot{v}(t)=-a_{21} y^{*} u\left(t-\tau_{21}(t)\right)-a_{22} y^{*} v\left(t-\tau_{22}(t)\right) .
\end{array}\right.
$$

Let $z^{T}(t)=\left(u^{T}(t), v^{T}(t)\right)$ and rewrite (4) in the following matrix form:

$$
\begin{aligned}
\dot{z}(t)= & -A_{11} z\left(t-\tau_{11}(t)\right)-A_{12} z\left(t-\tau_{12}(t)\right) \\
& -A_{21} z\left(t-\tau_{21}(t)\right)-A_{22} z\left(t-\tau_{22}(t)\right),
\end{aligned}
$$

where

$$
\begin{array}{ll}
A_{11}=\left[\begin{array}{cc}
a_{11} x^{*} & 0 \\
0 & 0
\end{array}\right], & A_{12}=\left[\begin{array}{cc}
0 & a_{12} x^{*} \\
0 & 0
\end{array}\right], \\
A_{21}=\left[\begin{array}{cc}
0 & 0 \\
a_{21} y^{*} & 0
\end{array}\right], & A_{22}=\left[\begin{array}{cc}
0 & 0 \\
0 & a_{22} y^{*}
\end{array}\right] .
\end{array}
$$

The following lemma of Wirtinger-based integral inequality plays an important role in the proof of our main results 
Lemma 1 ([26]) For a given matrix $R>0$, the following inequality holds for differentiable function $w(t)$ in $[a, b] \rightarrow \mathbb{R}^{n}$ :

$$
\int_{a}^{b} \dot{w}^{T}(u) R \dot{w}(u) \geq \frac{1}{b-a}(w(b)-w(a))^{T} R(w(b)-w(a))+\frac{3}{b-a} \Omega^{T} R \Omega,
$$

where $\Omega=w(b)+w(a)-\frac{2}{b-a} \int_{a}^{b} w(u) d u$.

\section{Main results}

In this section, by using the augmented L-K functionals and the Wirtinger-based integral inequality, one can obtain the following theorems for ensuring the local asymptotic stability of the L-V system (1). First of all, we will consider the case of constant delays, i.e., $\tau_{i j}(t)=\tau_{i j}(t \geq 0)$.

Theorem 1 The positive equilibrium $z^{*}$ of the L-V system (1) with constant delays is locally asymptotically stable, if there exist $10 \times 10$-dimensional matrix $P_{1}>0,2 \times 2$-dimensional matrices $Q_{i j} \geq 0, R_{i j}>0, i, j=1,2$, such that the following LMI holds:

$$
\psi_{1}=\mathbf{H e}\left(F_{1}^{T} P_{1} F_{0}\right)+\Gamma_{1}+\Upsilon_{1}<0
$$

where $\Gamma_{1}=\operatorname{diag}\left\{\sum_{i, j=1}^{2} Q_{i j}, 0,0,0,0,-Q_{11},-Q_{12},-Q_{21},-Q_{22}\right\}$ and

$$
\begin{aligned}
& F_{1}=\left[\begin{array}{lllllllll}
I & 0 & 0 & 0 & 0 & 0 & 0 & 0 & 0 \\
0 & I & 0 & 0 & 0 & 0 & 0 & 0 & 0 \\
0 & 0 & I & 0 & 0 & 0 & 0 & 0 & 0 \\
0 & 0 & 0 & I & 0 & 0 & 0 & 0 & 0 \\
0 & 0 & 0 & 0 & I & 0 & 0 & 0 & 0
\end{array}\right] \\
& F_{0}=\left[\begin{array}{ccccccccc}
0 & 0 & 0 & 0 & 0 & -A_{11} & -A_{12} & -A_{21} & -A_{22} \\
I & 0 & 0 & 0 & 0 & -I & 0 & 0 & 0 \\
I & 0 & 0 & 0 & 0 & 0 & -I & 0 & 0 \\
I & 0 & 0 & 0 & 0 & 0 & 0 & -I & 0 \\
I & 0 & 0 & 0 & 0 & 0 & 0 & 0 & -I
\end{array}\right], \\
& \Upsilon_{1}=\left[\begin{array}{ccccccccc}
\Lambda_{1} & \frac{6}{\tau_{11}} R_{11} & \frac{6}{\tau_{12}} R_{12} & \frac{6}{\tau_{21}} R_{21} & \frac{6}{\tau_{22}} R_{22} & -2 R_{11} & -2 R_{12} & -2 R_{21} & -2 R_{22} \\
* & -\frac{12}{\tau_{11}^{2}} R_{11} & 0 & 0 & 0 & \frac{6}{\tau_{11}} R_{11} & 0 & 0 & 0 \\
* & * & -\frac{12}{\tau_{12}^{2}} R_{12} & 0 & 0 & 0 & \frac{6}{\tau_{12}} R_{12} & 0 & 0 \\
* & * & * & -\frac{12}{\tau_{21}^{2}} R_{21} & 0 & 0 & 0 & \frac{6}{\tau_{21}} R_{21} & 0 \\
* & * & * & * & -\frac{12}{\tau_{22}^{2}} R_{22} & 0 & 0 & 0 & \frac{6}{\tau_{22}} R_{22} \\
* & * & * & * & * & \Lambda_{2} & A_{12}^{T} \Sigma A_{12} & A_{21}^{T} \Sigma A_{21} & A_{22}^{T} \Sigma A_{22} \\
* & * & * & * & * & * & \Lambda_{3} & A_{21}^{T} \Sigma A_{21} & A_{22}^{T} \Sigma A_{22} \\
* & * & * & * & * & * & * & \Lambda_{4} & A_{22}^{T} \Sigma A_{22} \\
* & * & * & * & * & * & * & * & \Lambda_{5}
\end{array}\right]
\end{aligned}
$$

with

$$
\begin{aligned}
& \Lambda_{1}=-4\left(R_{11}+R_{12}+R_{21}+R_{22}\right), \quad \Lambda_{2}=A_{11}^{T} \Sigma A_{11}-4 R_{11}, \\
& \Lambda_{3}=A_{12}^{T} \Sigma A_{12}-4 R_{12}, \quad \Lambda_{4}=A_{21}^{T} \Sigma A_{21}-4 R_{21}, \\
& \Lambda_{5}=A_{22}^{T} \Sigma A_{22}-4 R_{22}, \quad \Sigma=\tau_{11}^{2} R_{11}+\tau_{12}^{2} R_{12}+\tau_{21}^{2} R_{21}+\tau_{22}^{2} R_{22} .
\end{aligned}
$$


Proof Consider the following augmented L-K functional:

$$
\begin{aligned}
V(t)= & \bar{z}_{1}^{T}(t) P_{1} \bar{z}_{1}(t)+\sum_{i, j=1}^{2} \int_{t-\tau_{i j}}^{t} z^{T}(s) Q_{i j} z(s) d s \\
& +\sum_{i, j=1}^{2} \tau_{i j} \int_{t-\tau_{i j}}^{t} \int_{\theta}^{t} \dot{z}^{T}(s) R_{i j} \dot{z}(s) d s d \theta,
\end{aligned}
$$

where $\bar{z}_{1}(t)=\left[z^{T}(t), \int_{t-\tau_{11}}^{t} z^{T}(s) d s, \int_{t-\tau_{12}}^{t} z^{T}(s) d s, \int_{t-\tau_{21}}^{t} z^{T}(s) d s, \int_{t-\tau_{22}}^{t} z^{T}(s) d s\right]^{T}$.

Clearly, functional (8) is positive since $P_{1}>0, Q_{i j}>0$, and $R_{i j}>0$. Denoting that $\xi_{1}(t)=$ $\left[\bar{z}_{1}^{T}(t), z^{T}\left(t-\tau_{11}\right), z^{T}\left(t-\tau_{12}\right), z^{T}\left(t-\tau_{21}\right), z^{T}\left(t-\tau_{22}\right)\right]^{T}$, we have

$$
\bar{z}_{1}(t)=F_{1} \xi_{1}(t), \quad \dot{\bar{z}}_{1}(t)=F_{0} \xi_{1}(t) .
$$

Considering the derivative of $V(t)$ along system (5), we have

$$
\begin{aligned}
\dot{V}(t)= & \xi_{1}^{T}(t) \mathbf{H e}\left(F_{1}^{T} P_{1} F_{0}\right) \xi_{1}(t)+z^{T}(t)\left(\sum_{i, j=1}^{2} Q_{i j}\right) z(t) \\
& -\sum_{i, j=1}^{2} z^{T}\left(t-\tau_{i j}\right) Q_{i j} z\left(t-\tau_{i j}\right)+\dot{z}^{T}(t)\left(\sum_{i, j=1}^{2} \tau_{i j}^{2} R_{i j}\right) \dot{z}(t) \\
& -\sum_{i, j=1}^{2} \tau_{i j} \int_{t-\tau_{i j}}^{t} \dot{z}^{T}(s) R_{i j} \dot{z}(s) d s .
\end{aligned}
$$

By using Lemma 1, it follows that

$$
\begin{aligned}
\dot{V}(t) \leq & \xi_{1}^{T}(t) \mathbf{H e}\left(F_{1}^{T} P_{1} F_{0}\right) \xi_{1}(t)+z^{T}(t)\left(\sum_{i, j=1}^{2} Q_{i j}\right) z(t) \\
& -\sum_{i, j=1}^{2} z^{T}\left(t-\tau_{i j}\right) Q_{i j} z\left(t-\tau_{i j}\right) \\
& +\left[\sum_{i, j=1}^{2} A_{i j} z\left(t-\tau_{i j}\right)\right]^{T}\left(\sum_{i, j=1}^{2} \tau_{i j}^{2} R_{i j}\right)\left[\sum_{i, j=1}^{2} A_{i j} z\left(t-\tau_{i j}\right)\right] \\
& -\sum_{i, j=1}^{2}\left\{\left[z\left(t-\tau_{i j}\right)-z(t)\right]^{T} R_{i j}\left[z\left(t-\tau_{i j}\right)-z(t)\right]\right. \\
& -3\left[z\left(t-\tau_{i j}\right)+z(t)-\frac{2}{\tau_{i j}} \int_{t-\tau_{i j}}^{t} z(s) d s\right]^{T} R_{i j} \\
& \left.\times\left[z\left(t-\tau_{i j}\right)+z(t)-\frac{2}{\tau_{i j}} \int_{t-\tau_{i j}}^{t} z(s) d s\right]\right\} \\
= & \xi_{1}^{T}(t) \psi_{1} \xi_{1}(t),
\end{aligned}
$$

where $\psi_{1}$ is defined in (7). If LMI (7) is satisfied, we have $\dot{V}(t) \leq \xi_{1}^{T}(t) \psi_{1} \xi_{1}(t)<0$ and the proof of Theorem 1 is complete. 
Remark 1 Note that if LMI (7) is true, it follows that $\dot{V}(t) \leq-\lambda\|z(t)\|^{2}$, where $\lambda>0$ is the minimal eigenvalue of the matrix $-\psi_{1}$. This implies that system (5) is asymptotically stable. Then it can be concluded that the positive equilibrium $z^{*}$ of the L-V system (1) is locally asymptotically stable.

Remark 2 In [22-24], an LMI-based approach has been used to address the stability problem of systems (1), and some significant results have been obtained. However, it is worth mentioning that the proposed conditions are conservative since the chosen L-K functional is simple and the adopted inequalities are conservative. Compared with the approaches used in [22-24], this paper has utilized more accurate Wirtinger inequality to estimate the upper bound of the derivative of L-K functional (8). In particular, it is noted that some augmented terms have been added in the L-K functional (8) to use Wirtinger inequality effectively.

Theorem 2 The positive equilibrium $z^{*}$ of the L-V system (1) with constant delays is locally asymptotically stable if there exist $22 \times 22$-dimensional matrix $P_{2}>0,2 \times 2$-dimensional matrices $Q_{i j}^{k l} \geq 0$ and $R_{i j}^{k l}>0,(i, j, k, l=1,2)$ such that the following LMI holds:

$$
\psi_{2}=\mathbf{H e}\left(\tilde{F}_{1}^{T} P_{2} \tilde{F}_{0}\right)+\Gamma_{2}+\Upsilon_{2}<0
$$

where

$$
\begin{aligned}
& \tilde{F}_{1}=\left[\begin{array}{cc}
F_{1} & 0_{10 \times 12} \\
0_{12 \times 18} & \Xi
\end{array}\right], \quad \tilde{F}_{0}=\left[\begin{array}{cc}
F_{0} & 0_{10 \times 12} \\
\tilde{\Omega} & \Xi
\end{array}\right], \\
& \Gamma_{2}=\left[\begin{array}{cc}
\Gamma_{1} & 0_{18 \times 12} \\
0_{12 \times 18} & 0_{12 \times 12}
\end{array}\right]+\operatorname{diag}\left\{0_{10 \times 10}, \bar{Q}, 0_{12 \times 12}\right\}, \\
& \Upsilon_{2}=\left[\begin{array}{cc}
\Upsilon_{1} & 0_{18 \times 12} \\
0_{12 \times 18} & 0_{12 \times 12}
\end{array}\right]+\left[\begin{array}{ccc}
0_{10 \times 10} & 0_{10 \times 8} & 0_{10 \times 12} \\
* & \Phi_{1}-2 \Phi_{2} & 6 \Phi_{3} \\
* & * & -12 \Phi_{4}
\end{array}\right],
\end{aligned}
$$

with $F_{0}, F_{1}, \Gamma_{1}, \Upsilon_{1}$ denoted in Theorem 1 and

$$
\begin{aligned}
\tilde{\Omega}= & {\left[\begin{array}{ccccccccc}
0 & 0 & 0 & 0 & 0 & -I & I & 0 & 0 \\
0 & 0 & 0 & 0 & 0 & -I & 0 & I & 0 \\
0 & 0 & 0 & 0 & 0 & -I & 0 & 0 & I \\
0 & 0 & 0 & 0 & 0 & 0 & -I & I & 0 \\
0 & 0 & 0 & 0 & 0 & 0 & -I & 0 & I \\
0 & 0 & 0 & 0 & 0 & 0 & 0 & -I & I
\end{array}\right], } \\
\bar{Q}= & -\operatorname{diag}\left\{\sum_{k, l=1}^{2}\left(\tau_{11}-\tau_{k l}\right) Q_{11}^{k l}, \sum_{k, l=1}^{2}\left(\tau_{12}-\tau_{k l}\right) Q_{12}^{k l},\right. \\
& \left.\sum_{k, l=1}^{2}\left(\tau_{21}-\tau_{k l}\right) Q_{21}^{k l}, \sum_{k, l=1}^{2}\left(\tau_{22}-\tau_{k l}\right) Q_{22}^{k l}\right\} \\
\Xi= & \operatorname{diag}\left\{\left(\tau_{11}-\tau_{12}\right) I,\left(\tau_{11}-\tau_{21}\right) I,\left(\tau_{11}-\tau_{22}\right) I,\right. \\
& \left.\left(\tau_{12}-\tau_{21}\right) I,\left(\tau_{12}-\tau_{22}\right) I,\left(\tau_{21}-\tau_{22}\right) I\right\}
\end{aligned}
$$




$$
\begin{aligned}
& \Phi_{1}=\left[\begin{array}{cccc}
A_{11}^{T} \tilde{\Sigma} A_{11} & A_{11}^{T} \tilde{\Sigma} A_{12} & A_{11}^{T} \tilde{\Sigma} A_{21} & A_{11}^{T} \tilde{\Sigma} A_{22} \\
* & A_{12}^{T} \tilde{\Sigma} A_{12} & A_{12}^{T} \tilde{\Sigma} A_{21} & A_{12}^{T} \tilde{\Sigma} A_{22} \\
* & * & A_{21}^{T} \tilde{\Sigma} A_{21} & A_{21}^{T} \tilde{\Sigma} A_{22} \\
* & * & * & A_{22}^{T} \tilde{\Sigma} A_{22}
\end{array}\right], \\
& \Phi_{2}=\left[\begin{array}{cccc}
\Phi_{21} & R_{11}^{12} & R_{11}^{21} & R_{11}^{22} \\
* & \Phi_{22} & R_{12}^{21} & R_{12}^{22} \\
* & * & \Phi_{23} & R_{21}^{22} \\
* & * & * & \Phi_{24}
\end{array}\right] \text {, } \\
& \Phi_{3}=\left[\begin{array}{cccccc}
R_{11}^{12} & R_{11}^{21} & R_{11}^{22} & 0 & 0 & 0 \\
R_{11}^{12} & 0 & 0 & R_{12}^{21} & R_{12}^{22} & 0 \\
0 & R_{11}^{21} & 0 & R_{12}^{21} & 0 & R_{21}^{22} \\
0 & 0 & R_{11}^{22} & 0 & R_{12}^{22} & R_{21}^{22}
\end{array}\right] \text {, } \\
& \Phi_{4}=\operatorname{diag}\left\{R_{11}^{12}, R_{11}^{21}, R_{11}^{22}, R_{12}^{21}, R_{12}^{22}, R_{21}^{22}\right\} \text {, } \\
& \tilde{\Sigma}=\sum_{i, j=1}^{2} \sum_{k, l=1}^{2}\left(\tau_{i j}-\tau_{k l}\right)^{2} R_{i j}^{k l} \quad(10 i+j<10 k+l), \\
& \Phi_{21}=2\left(R_{11}^{12}+R_{11}^{21}+R_{11}^{22}\right), \quad \Phi_{22}=2\left(R_{11}^{12}+R_{12}^{21}+R_{12}^{22}\right), \\
& \Phi_{23}=2\left(R_{11}^{21}+R_{12}^{21}+R_{21}^{22}\right), \quad \Phi_{24}=2\left(R_{11}^{22}+R_{12}^{22}+R_{21}^{22}\right) .
\end{aligned}
$$

Proof Let us define the following novel augmented L-K functional:

$$
\tilde{V}(t)=V_{1}(t)+V_{2}(t)+V_{3}(t)
$$

where

$$
\begin{aligned}
V_{1}(t)= & \bar{z}_{2}^{T}(t) P_{2} \bar{z}_{2}(t)+\sum_{i, j=1}^{2} \int_{t-\tau_{i j}}^{t} z^{T}(s) Q_{i j} z(s) d s \\
& +\sum_{i, j=1}^{2} \tau_{i j} \int_{t-\tau_{i j}}^{t} \int_{\theta}^{t} \dot{z}^{T}(s) R_{i j} \dot{z}(s) d s d \theta, \\
V_{2}(t)= & \sum_{i, j=1}^{2} \sum_{k, l=1}^{2}\left(\tau_{i j}-\tau_{k l}\right) \int_{t-\tau_{i j}}^{t-\tau_{k l}} z^{T}(s) Q_{i j}^{k l} z(s) d s \quad(10 i+j<10 k+l), \\
V_{3}(t)= & \sum_{i, j=1}^{2} \sum_{k, l=1}^{2}\left(\tau_{i j}-\tau_{k l}\right) \int_{-\tau_{i j}}^{-\tau_{k l}} \int_{t+\theta}^{t} \dot{z}^{T}(s) R_{i j}^{k l} \dot{z}(s) d s d \theta \\
& (10 i+j<10 k+l),
\end{aligned}
$$

with

$$
\begin{aligned}
\tilde{z}_{2}(t)= & {\left[\bar{z}_{1}^{T}(t), \int_{t-\tau_{11}}^{t-\tau_{12}} z^{T}(s) d s, \int_{t-\tau_{11}}^{t-\tau_{21}} z^{T}(s) d s, \int_{t-\tau_{11}}^{t-\tau_{22}} z^{T}(s) d s,\right.} \\
& \left.\int_{t-\tau_{12}}^{t-\tau_{21}} z^{T}(s) d s, \int_{t-\tau_{12}}^{t-\tau_{22}} z^{T}(s) d s, \int_{t-\tau_{21}}^{t-\tau_{22}} z^{T}(s) d s\right]^{T} .
\end{aligned}
$$


Letting

$$
\begin{aligned}
\xi_{2}(t)= & {\left[\xi_{1}^{T}(t), \frac{1}{\tau_{11}-\tau_{12}} \int_{t-\tau_{11}}^{t-\tau_{12}} z^{T}(s) d s, \frac{1}{\tau_{11}-\tau_{21}} \int_{t-\tau_{11}}^{t-\tau_{21}} z^{T}(s) d s,\right.} \\
& \frac{1}{\tau_{11}-\tau_{22}} \int_{t-\tau_{11}}^{t-\tau_{22}} z^{T}(s) d s, \frac{1}{\tau_{12}-\tau_{21}} \int_{t-\tau_{12}}^{t-\tau_{21}} z^{T}(s) d s, \\
& \left.\frac{1}{\tau_{12}-\tau_{22}}, \int_{t-\tau_{12}}^{t-\tau_{22}} z^{T}(s) d s, \frac{1}{\tau_{21}-\tau_{22}} \int_{t-\tau_{21}}^{t-\tau_{22}} z^{T}(s) d s\right]^{T},
\end{aligned}
$$

it is seen that $\tilde{z}_{2}(t)=\tilde{F}_{1} \xi_{2}(t), \dot{\tilde{z}}_{2}(t)=\tilde{F}_{0} \xi_{2}(t)$. Computing the derivative of (12) along the solution of system (5), it follows that

$$
\begin{aligned}
\dot{V}_{1}(t)= & \xi_{2}^{T}(t) \mathbf{H e}\left(\tilde{F}_{0}^{T} P_{2} \tilde{F}_{1}\right) \xi_{2}(t)+z^{T}(t)\left(\sum_{i, j=1}^{2} Q_{i j}\right) z(t) \\
& -\sum_{i, j=1}^{2} z^{T}\left(t-\tau_{i j}\right) Q_{i j} z\left(t-\tau_{i j}\right)+\dot{z}^{T}(t)\left(\sum_{i, j=1}^{2} \tau_{i j}^{2} R_{i j}\right) \dot{z}(t) \\
& -\sum_{i, j=1}^{2} \tau_{i j} \int_{t-\tau_{i j}}^{t} \dot{z}^{T}(s) R_{i j} \dot{z}(s) d s, \\
\dot{V}_{2}(t)= & \sum_{i, j=1}^{2} \sum_{k, l=1}^{2}\left(\tau_{i j}-\tau_{k l}\right)\left[z^{T}\left(t-\tau_{k l}\right) Q_{i j}^{k l} z\left(t-\tau_{k l}\right)\right. \\
& \left.-z^{T}\left(t-\tau_{i j}\right) Q_{i j}^{k l} z\left(t-\tau_{i j}\right)\right] \quad(10 i+j<10 k+l), \\
\dot{V}_{3}(t)= & \sum_{i, j=1}^{2} \sum_{k, l=1}^{2}\left(\tau_{i j}-\tau_{k l}\right)\left[\left(\tau_{i j}-\tau_{k l}\right) \dot{z}^{T}(t) R_{i j}^{k l} \dot{z}(t)\right. \\
& \left.-\int_{t-\tau_{i j}}^{t-\tau_{k l}} \dot{z}^{T}(s) R_{i j}^{k l} \dot{z}(s) d s\right] \quad(10 i+j<10 k+l) .
\end{aligned}
$$

Applying Lemma 1 to $\dot{V}_{3}(t)$, it follows that

$$
\begin{aligned}
\dot{V}_{3}(t) \leq & \sum_{i, j=1}^{2} \sum_{k, l=1}^{2}\left\{\left(\tau_{i j}-\tau_{k l}\right)^{2} \dot{z}^{T}(t) R_{i j}^{k l} \dot{z}(t)\right. \\
& -\left[z\left(t-\tau_{k l}\right)-z\left(t-\tau_{i j}\right)\right]^{T} R_{i j}^{k l}\left[z\left(t-\tau_{k l}\right)-z\left(t-\tau_{i j}\right)\right] \\
& -3\left[z\left(t-\tau_{k l}\right)+z\left(t-\tau_{i j}\right)-\frac{2}{\left(\tau_{i j}-\tau_{k l}\right)} \int_{t-\tau_{i j}}^{t-\tau_{k l}} z(s) d s\right]^{T} R_{i j}^{k l} \\
& \left.\times\left[z\left(t-\tau_{k l}\right)+z\left(t-\tau_{i j}\right)-\frac{2}{\left(\tau_{i j}-\tau_{k l}\right)} \int_{t-\tau_{i j}}^{t-\tau_{k l}} z(s) d s\right]\right\} \\
& (10 i+j<10 k+l) .
\end{aligned}
$$

Based on $\dot{V}_{1}(t), \dot{V}_{2}(t), \dot{V}_{3}(t)$, and inequality (16), we can eventually obtain

$$
\dot{\tilde{V}}(t)=\dot{V}_{1}(t)+\dot{V}_{2}(t)+\dot{V}_{3}(t) \leq \xi_{2}^{T}(t) \psi_{2} \xi_{2}(t) .
$$


This implies that the positive equilibrium $z^{*}$ of the L-V system (1) is locally asymptotically stable if LMI (11) is satisfied. The proof of Theorem 2 is complete.

Remark 3 Compared with the L-K functional (8) used in the proof of Theorem 1, some interconnected terms related to four time delays have been further incorporated in functional (12). By using such interconnected terms, the relationships between four time delays can be specifically reflected in the L-K functional (12). Then, a less conservative condition involving more slack variables is obtained for the case with different delays, which will be illustrated by a numerical example.

Next, we will address the L-V system (1) with time-varying delays.

Theorem 3 The positive equilibrium $z^{*}$ of the L-V system (1) with time-varying delays is locally asymptotically stable if there exist $10 \times 10$-dimensional matrix $P_{1}>0,2 \times 2$ dimensional matrices $Q_{i j} \geq 0, R_{i j}>0, S_{i j}>0, T_{i j}>0, i, j=1,2$, such that the following $L M I$ holds:

$$
\begin{aligned}
\psi_{3}= & {\left[\begin{array}{cc}
\tilde{\psi}_{1} & 0_{18 \times 8} \\
0_{8 \times 18} & \Upsilon_{3}
\end{array}\right]+\Gamma_{3}+\mathbf{H e}\left(\hat{F}_{1}^{T} P_{1} \hat{F}_{0}\right) } \\
& +\left[\begin{array}{cccc}
-\sum_{i, j=1}^{2} T_{i j} & 0_{2 \times 8} & 0_{2 \times 8} & \Pi_{1} \\
* & 0_{8 \times 8} & 0_{8 \times 8} & 0_{8 \times 8} \\
* & * & -\Pi_{2} & \Pi_{2} \\
* & * & * & -2 \Pi_{2}
\end{array}\right] \\
<0 &
\end{aligned}
$$

where

$$
\begin{aligned}
& \Gamma_{3}=\operatorname{diag}\left\{\sum_{i, j=1}^{2} S_{i j}, 0_{16 \times 16},-\left(1-d_{11}\right) S_{11},-\left(1-d_{12}\right) S_{12}\right. \text {, } \\
& \left.-\left(1-d_{21}\right) S_{21},-\left(1-d_{22}\right) S_{22}\right\} \\
& \hat{F}_{0}=\left[\begin{array}{cccc}
0_{2 \times 2} & 0_{2 \times 8} & 0_{2 \times 8} & \hat{F}_{02} \\
\hat{F}_{01} & 0_{8 \times 8} & \hat{F}_{03} & 0_{8 \times 8}
\end{array}\right], \quad \hat{F}_{1}=\left[\begin{array}{ll}
F_{1} & 0_{10 \times 8}
\end{array}\right] \text {, } \\
& \tilde{\psi}_{1}=\psi_{1}-\mathbf{H e}\left(F_{1}^{T} P_{1} F_{0}\right)-\left[\begin{array}{cc}
0_{10 \times 10} & 0_{10 \times 8} \\
0_{8 \times 10} & \Pi_{4}
\end{array}\right], \\
& \Upsilon_{3}=\left[\begin{array}{cccc}
A_{11}^{T} \hat{\Sigma} A_{11} & A_{11}^{T} \hat{\Sigma} A_{12} & A_{11}^{T} \hat{\Sigma} A_{21} & A_{11}^{T} \hat{\Sigma} A_{22} \\
* & A_{12}^{T} \hat{\Sigma} A_{12} & A_{12}^{T} \hat{\Sigma} A_{21} & A_{12}^{T} \hat{\Sigma} A_{22} \\
* & * & A_{21}^{T} \hat{\Sigma} A_{21} & A_{21}^{T} \hat{\Sigma} A_{22} \\
* & * & * & A_{22}^{T} \hat{\Sigma} A_{22}
\end{array}\right] \text {, } \\
& \Pi_{1}=\left[\begin{array}{llll}
T_{11} & T_{12} & T_{21} & T_{22}
\end{array}\right], \quad \Pi_{2}=\operatorname{diag}\left\{T_{11}, T_{12}, T_{21}, T_{22}\right\}
\end{aligned}
$$

with

$$
\hat{F}_{01}=\left[\begin{array}{llll}
I & I & I & I
\end{array}\right]^{T}, \quad \hat{F}_{02}=\left[\begin{array}{llll}
-A_{11} & -A_{12} & -A_{21} & -A_{11}
\end{array}\right],
$$




$$
\begin{aligned}
& \hat{F}_{03}=-\operatorname{diag}\{I, I, I, I\}, \quad \hat{\Sigma}=\sum_{i, j=1}^{2}\left[\tau_{i j}^{2}\left(T_{i j}+R_{i j}\right)\right], \\
& \Pi_{4}=\left[\begin{array}{cccc}
A_{11}^{T} \Sigma A_{11} & A_{11}^{T} \Sigma A_{12} & A_{11}^{T} \Sigma A_{21} & A_{11}^{T} \Sigma A_{22} \\
* & A_{12}^{T} \Sigma A_{12} & A_{12}^{T} \Sigma A_{21} & A_{12}^{T} \Sigma A_{22} \\
* & * & A_{21}^{T} \Sigma A_{21} & A_{21}^{T} \Sigma A_{22} \\
& * & * & A_{22}^{T} \Sigma A_{22}
\end{array}\right] .
\end{aligned}
$$

Proof Consider the following augmented L-K functional:

$$
\begin{aligned}
\hat{V}(t)= & V(t)+\sum_{i, j=1}^{2} \int_{t-\tau_{i j}(t)}^{t} z^{T}(s) S_{i j} z(s) d s \\
& +\sum_{i, j=1}^{2} \tau_{i j} \int_{t-\tau_{i j}}^{t} \int_{\theta}^{t} \dot{z}^{T}(s) T_{i j} \dot{z}(s) d s d \theta,
\end{aligned}
$$

where $V(t)$ is denoted in (8). Considering the derivative of $\hat{V}(t)$ and denoting $\xi_{3}(t)=$ $\left[\xi_{1}^{T}(t), z^{T}\left(t-\tau_{11}(t)\right), z^{T}\left(t-\tau_{12}(t)\right), z^{T}\left(t-\tau_{21}(t)\right), z^{T}\left(t-\tau_{22}(t)\right)\right]^{T}$, we have

$$
\begin{aligned}
\dot{\hat{V}}(t) \leq & \xi_{3}^{T}(t) \mathbf{H e}\left(\hat{F}_{1}^{T} P_{1} \hat{F}_{0}\right) \xi_{3}(t)+z^{T}(t) \sum_{i, j=1}^{2}\left(Q_{i j}+S_{i j}\right) z(t) \\
& +\dot{z}^{T}(t) \sum_{i, j=1}^{2} \tau_{i j}^{2}\left(R_{i j}+T_{i j}\right) \dot{z}(t)-\sum_{i, j=1}^{2} z^{T}\left(t-\tau_{i j}\right) Q_{i j} z\left(t-\tau_{i j}\right) \\
& -\sum_{i, j=1}^{2}\left(1-d_{i j}\right) z^{T}\left(t-\tau_{i j}(t)\right) S_{i j} z\left(t-\tau_{i j}(t)\right) \\
& -\sum_{i, j=1}^{2} \tau_{i j} \int_{t-\tau_{i j}}^{t} \dot{z}^{T}(s)\left(R_{i j}+T_{i j}\right) \dot{z}(s) d s .
\end{aligned}
$$

For the term $-\sum_{i, j=1}^{2} \tau_{i j} \int_{t-\tau_{i j}}^{t} \dot{z}^{T}(s) R_{i j} \dot{z}(s) d s$, we use Lemma 1. While for the term $-\sum_{i, j=1}^{2} \tau_{i j} \int_{t-\tau_{i j}}^{t} \dot{z}^{T}(s) T_{i j} \dot{z}(s) d s$, by using Jensen's inequality, it is seen that

$$
\begin{aligned}
& -\sum_{i, j=1}^{2} \tau_{i j} \int_{t-\tau_{i j}}^{t} \dot{z}^{T}(s) T_{i j} \dot{z}(s) d s \\
& \leq-\sum_{i, j=1}^{2}\left(\int_{t-\tau_{i j}(t)}^{t} \dot{z}(s) d s\right)^{T} T_{i j}\left(\int_{t-\tau_{i j}(t)}^{t} \dot{z}(s) d s\right) \\
& \quad-\sum_{i, j=1}^{2}\left(\int_{t-\tau_{i j}}^{t-\tau_{i j}(t)} \dot{z}(s) d s\right)^{T} T_{i j}\left(\int_{t-\tau_{i j}}^{t-\tau_{i j}(t)} \dot{z}(s) d s\right) \\
& \leq-\sum_{i, j=1}^{2}\left[z(t)-z\left(t-\tau_{i j}(t)\right)\right]^{T} T_{i j}\left[z(t)-z\left(t-\tau_{i j}(t)\right)\right] \\
& \quad-\sum_{i, j=1}^{2}\left[z\left(t-\tau_{i j}(t)\right)-z\left(t-\tau_{i j}\right)\right]^{T} T_{i j}\left[z\left(t-\tau_{i j}(t)\right)-z\left(t-\tau_{i j}\right)\right] .
\end{aligned}
$$


Noting (20)-(21) and $\psi_{3}$ defined in (18), it is easy to verify that if LMI (18) is satisfied, the inequality $\dot{\hat{V}}(t) \leq \xi_{3}^{T}(t) \psi_{3} \xi_{3}(t)<0$ can be ensured, and this completes the proof.

Corresponding to Theorem 2, the following improved condition is readily obtained.

Theorem 4 The positive equilibrium $z^{*}$ of the $L$-V system (1) with time-varying delays is locally asymptotically stable if there exist $22 \times 22$-dimensional matrix $P_{2}>0,2 \times 2$ dimensional matrices $Q_{i j} \geq 0, R_{i j}>0, S_{i j}>0, T_{i j}>0, i, j=1,2$, such that the following LMI holds:

$$
\begin{aligned}
\psi_{4}= & {\left[\begin{array}{cc}
\tilde{\psi}_{2} & 0_{30 \times 8} \\
0_{8 \times 30} & \Upsilon_{4}
\end{array}\right]+\Gamma_{4}+\mathbf{H e}\left(\vec{F}_{1}^{T} P_{2} \vec{F}_{0}\right) } \\
& +\left[\begin{array}{ccccc}
0_{2 \times 2} & 0_{2 \times 8} & 0_{2 \times 8} & 0_{2 \times 12} & \Upsilon_{5} \\
* & 0_{8 \times 8} & 0_{8 \times 8} & 0_{8 \times 12} & 0_{8 \times 8} \\
* & * & -\Upsilon_{6} & 0_{8 \times 12} & \Upsilon_{6} \\
* & * & * & 0_{12 \times 12} & 0_{12 \times 8} \\
* & * & * & * & -2 \Upsilon_{6}
\end{array}\right] \\
<0, &
\end{aligned}
$$

where

$$
\begin{aligned}
& \Gamma_{4}=\operatorname{diag}\left\{\sum_{i, j=1}^{2}\left(S_{i j}-T_{i j}\right), 0_{28 \times 28},-\left(1-d_{11}\right) S_{11}\right. \text {, } \\
& \left.-\left(1-d_{12}\right) S_{12},-\left(1-d_{21}\right) S_{21},-\left(1-d_{22}\right) S_{22}\right\} \text {, } \\
& \vec{F}_{0}=\left[\begin{array}{ccccc}
0_{2 \times 2} & 0_{2 \times 8} & 0_{2 \times 8} & 0_{2 \times 12} & \hat{F}_{02} \\
\hat{F}_{01} & 0_{8 \times 8} & \hat{F}_{03} & 0_{8 \times 12} & 0_{8 \times 8} \\
0_{12 \times 2} & 0_{12 \times 8} & \hat{F}_{04} & \Xi & 0_{12 \times 8}
\end{array}\right], \quad \vec{F}_{1}=\left[\begin{array}{cc}
\tilde{F}_{1} & 0_{22 \times 8}
\end{array}\right], \\
& \tilde{\psi}_{2}=\psi_{2}-\mathbf{H e}\left(\tilde{F}_{1}^{T} P_{2} \tilde{F}_{0}\right)-\left[\begin{array}{ccc}
0_{10 \times 10} & 0_{10 \times 8} & 0_{10 \times 12} \\
0_{8 \times 10} & \Upsilon_{7} & 0_{8 \times 12} \\
0_{12 \times 10} & 0_{12 \times 8} & 0_{12 \times 12}
\end{array}\right], \\
& \Upsilon_{4}=\left[\begin{array}{cccc}
A_{11}^{T} \vec{\Sigma}_{1} A_{11} & A_{11}^{T} \vec{\Sigma}_{1} A_{12} & A_{11}^{T} \vec{\Sigma}_{1} A_{21} & A_{11}^{T} \vec{\Sigma}_{1} A_{22} \\
* & A_{12}^{T} \vec{\Sigma}_{1} A_{12} & A_{12}^{T} \vec{\Sigma}_{1} A_{21} & A_{12}^{T} \vec{\Sigma}_{1} A_{22} \\
* & * & A_{21}^{T} \vec{\Sigma}_{1} A_{21} & A_{21}^{T} \vec{\Sigma}_{1} A_{22} \\
* & * & * & A_{22}^{T} \vec{\Sigma}_{1} A_{22}
\end{array}\right] \\
& \Upsilon_{5}=\left[\begin{array}{llll}
T_{11} & T_{12} & T_{21} & T_{22}
\end{array}\right], \quad \Upsilon_{6}=\operatorname{diag}\left\{T_{11}, T_{12}, T_{21}, T_{22}\right\}
\end{aligned}
$$

with

$$
\hat{F}_{04}=\left[\begin{array}{cccccc}
-I & -I & -I & 0 & 0 & 0 \\
I & 0 & 0 & -I & -I & 0 \\
0 & I & 0 & I & 0 & -I \\
0 & 0 & I & 0 & I & I
\end{array}\right]^{T}
$$




$$
\begin{aligned}
& \Upsilon_{7}=\left[\begin{array}{cccc}
A_{11}^{T} \vec{\Sigma}_{2} A_{11} & A_{11}^{T} \vec{\Sigma}_{2} A_{12} & A_{11}^{T} \vec{\Sigma}_{2} A_{21} & A_{11}^{T} \vec{\Sigma}_{2} A_{22} \\
* & A_{12}^{T} \vec{\Sigma}_{2} A_{12} & A_{12}^{T} \vec{\Sigma}_{2} A_{21} & A_{12}^{T} \vec{\Sigma}_{2} A_{22} \\
* & * & A_{21}^{T} \vec{\Sigma}_{2} A_{21} & A_{21}^{T} \vec{\Sigma}_{2} A_{22} \\
* & * & * & A_{22}^{T} \vec{\Sigma}_{2} A_{22}
\end{array}\right], \\
& \vec{\Sigma}_{1}=\sum_{i, j=1}^{2} \tau_{i j}^{2}\left(T_{i j}+R_{i j}\right)+\sum_{i, j=1}^{2} \sum_{k, l=1}^{2}\left(\tau_{i j}-\tau_{k l}\right)^{2} R_{i j}^{k l} \quad(10 i+j<10 k+l) \text {, } \\
& \vec{\Sigma}_{2}=\sum_{i, j=1}^{2} \tau_{i j}^{2} R_{i j}+\sum_{i, j=1}^{2} \sum_{k, l=1}^{2}\left(\tau_{i j}-\tau_{k l}\right)^{2} R_{i j}^{k l} \quad(10 i+j<10 k+l) .
\end{aligned}
$$

Proof Let us define the following novel augmented L-K functional:

$$
\begin{aligned}
\vec{V}(t)= & \tilde{V}(t)+\sum_{i, j=1}^{2} \int_{t-\tau_{i j}(t)}^{t} z^{T}(s) S_{i j} z(s) d s \\
& +\sum_{i, j=1}^{2} \tau_{i j} \int_{t-\tau_{i j}}^{t} \int_{\theta}^{t} \dot{z}^{T}(s) T_{i j} \dot{z}(s) d s d \theta,
\end{aligned}
$$

where $\tilde{V}(t)$ is defined in functional (12). The subsequent arguments are similar to the proofs of Theorems $2-3$ and thus are omitted here.

\section{Numerical example}

In this section, let us consider the numerical example given in Zhen and Ma [21] and Park [22] to illustrate the effectiveness and the sharpness of our results.

Example Consider the following system:

$$
\left\{\begin{array}{l}
\frac{d x(t)}{d t}=x(t)\left[1-x\left(t-\tau_{11}(t)\right)-0.5 y\left(t-\tau_{12}(t)\right)\right] \\
\frac{d y(t)}{d t}=y(t)\left[1-0.5 x\left(t-\tau_{21}(t)\right)-y\left(t-\tau_{22}(t)\right)\right]
\end{array}\right.
$$

For the case that $\tau_{i j}(t) \equiv \tau_{i j}$, according to the result of [21], the equilibrium $(2 / 3,2 / 3)$ of system (24) is asymptotically stable if the following conditions hold:

$$
\begin{aligned}
& 28 \tau_{11}+8 \tau_{22}+5 \tau_{12}+13 \tau_{21}<18 \\
& 8 \tau_{11}+28 \tau_{22}+13 \tau_{12}+5 \tau_{21}<18
\end{aligned}
$$

When $\tau_{i j}=\tau$, the upper bounds $\tau$ for local asymptotic stability obtained by the existing results and the theorems in this paper are listed in Table 1 . For the case that $\tau_{11}=\tau_{12}=\tau_{21}$, we can easily obtain the maximum upper bounds of $\tau_{22}$ for various $\tau$, which are listed in Table 2. For the case of time-varying delay with $\tau_{i j}(t)=\tau(t) \leq \tau$ and $\dot{\tau}(t) \leq d$, by solving LMIs (18) and (22), the maximum admissible ranges of $\tau$ are obtained for different $d$,

Table 1 Maximum admissible ranges of the time delay $\tau$

\begin{tabular}{lllll}
\hline$[21]$ & {$[22]$} & {$[24]$} & Theorem 1 & Theorem 2 \\
\hline $0<\tau<0.34$ & $0<\tau<0.88$ & $0<\tau \leq 1.4307$ & $0<\tau \leq 1.5674$ & $0<\tau \leq 1.5674$ \\
\hline
\end{tabular}


Table 2 Maximum admissible upper bounds of $\tau_{22}$ for different $\tau$

\begin{tabular}{llllllllll}
\hline & \multicolumn{1}{l}{$\tau_{11}=\tau_{12}=\tau_{21}=\tau$} \\
\cline { 2 - 10 } & 0.5 & 0.7 & 0.9 & 1.0 & 1.1 & 1.2 & 1.3 & 1.4 & 1.5 \\
\hline$[22]$ & 1.28 & 1.11 & 0.79 & 0.50 & 0.02 & - & - & - & - \\
{$[24]$} & 1.86 & 1.74 & 1.60 & 1.53 & 1.47 & 1.40 & 1.31 & 1.21 & 1.04 \\
Theorem 1 & 2.71 & 2.80 & 2.87 & 2.87 & 2.83 & 2.74 & 2.59 & 2.28 & 1.80 \\
Theorem 2 & 2.72 & 2.84 & 2.95 & 3.01 & 3.06 & 3.12 & 3.18 & 3.23 & 3.27 \\
\hline
\end{tabular}

Table 3 Maximum admissible ranges of $\tau$ for different $d$

\begin{tabular}{llllllll}
\hline & \multicolumn{2}{l}{$d_{11}=d_{12}=d_{21}=d_{22}=d$} & & & \\
\cline { 2 - 8 } & 0 & 0.1 & 0.3 & 0.5 & 0.7 & 0.9 & $\geq 1$ \\
\hline [24] & 1.43 & 1.37 & 1.25 & 1.06 & 0.90 & 0.87 & 0.87 \\
Theorem 3 & 1.56 & 1.52 & 1.42 & 1.30 & 1.13 & 0.95 & 0.94 \\
Theorem 4 & 1.56 & 1.52 & 1.43 & 1.32 & 1.21 & 1.20 & 1.20 \\
\hline
\end{tabular}

Figure 1 State responses of system $(24)\left(\tau_{i j}=1.5\right)$

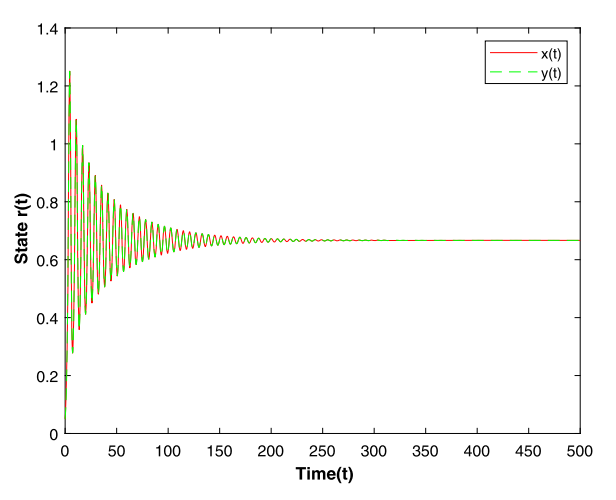

Figure 2 State responses of system (24)

$\left(\tau_{11}=\tau_{12}=\tau_{21}=1.5, \tau_{22}=1.8\right)$

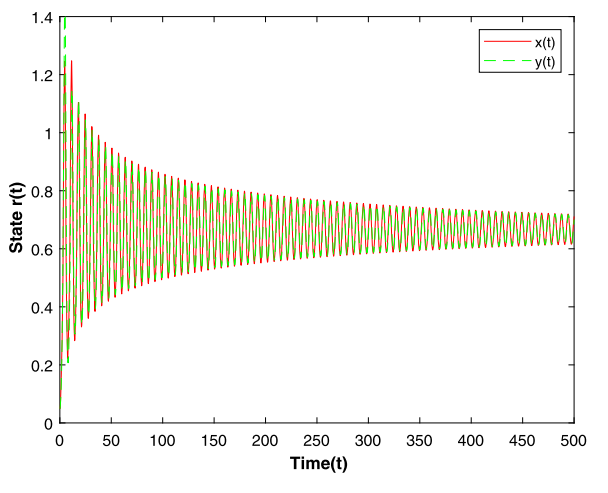

which are shown in Table 3. From Tables $1-3$, it is seen that our proposed results can provide the larger delay bounds than those in [21,22] and [24]. On the other hand, it is clear from Table 2 and Table 3 that Theorem 2 and Theorem 4 can provide larger delay bounds than Theorem 1 and Theorem 3, which means that the idea of using the relationships between four time delays is really effective in reducing the potential conservatism for the case with different delays. In the simulation, the state responses are plotted in Figs. 1-3. From Figs. $1-3$, it is clear that the equilibrium $(x *, y *)=(2 / 3,2 / 3)$ of system $(24)$ is asymptotically stable for the estimated ranges of time delays. 
Figure 3 State responses of system (24)

$\left(\tau_{11}=\tau_{12}=\tau_{21}=1.5, \tau_{22}=3.2\right)$

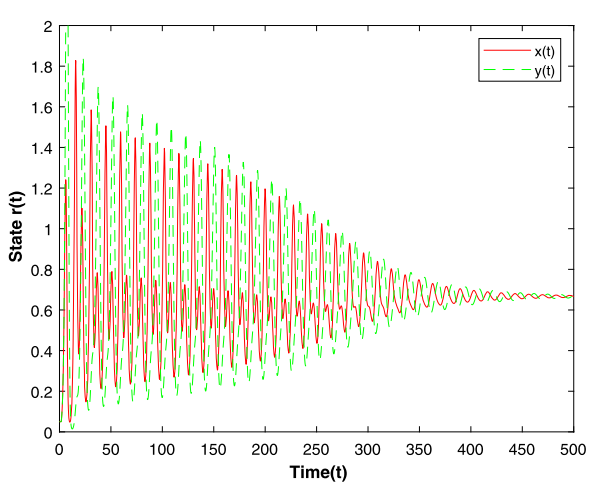

\section{Conclusions}

In this paper, we have addressed the local stability problem for a class of competitive L-V systems with time delays. By constructing some new quadratic L-K functionals and using the advanced Wirtinger integral inequality, four less conservative stability conditions have been obtained by means of LMIs. Different from most existing L-K functionals, some augmented terms have been introduced in our proposed L-K functionals. Moreover, the interconnected terms related to different time delays have been incorporated in the proposed L-K functional. Finally, a numerical example has been given to show the effectiveness and benefits of our obtained stability conditions. On the other hand, it is worth pointing out that the approach dealing with time-varying delays in this paper is standard. If some very recently developed techniques are employed [27-33], more effective conditions can be established.

\section{Acknowledgements}

The authors would like to thank the editors and the anonymous reviewers for their constructive comments which have improved the quality of the current manuscript.

Funding

This work was supported in part by the National Natural Science Foundation of China under Grant 61773156.

Availability of data and materials

Not applicable.

Competing interests

The authors declare that they have no competing interests.

Authors' contributions

All authors contributed equally to this paper. All authors read and approved the final manuscript.

\section{Publisher's Note}

Springer Nature remains neutral with regard to jurisdictional claims in published maps and institutional affiliations.

Received: 9 October 2018 Accepted: 16 April 2019 Published online: 09 May 2019

\section{References}

1. Volterra, V.: Leçons sur la théorie mathematique de la lutte pour la vie. Gauthier-Villars, Paris (1931)

2. Lotka, A.J.: Elements of Mathematical Biology. Dover, New York (1956)

3. Teng, Z:: Some new results of nonautonomous Lotka-Volterra competitive systems with delays. J. Math. Anal. Appl. $241(2), 254-275(2000)$

4. Schoener, T.W.: Field experiments on interspecific competition. Am. Nat. 122(2), 240-285 (1983)

5. Yang, K.: Delay Differential Equations with Applications in Population Dynamics. Academic Press, New York (1993)

6. Ahmad, S., Stamova, I.M.: Partial persistence and extinction in N-dimensional competitive systems. Nonlinear Anal., Real World Appl. 60(5), 821-836 (2015)

7. Han, Q.-L.: A discrete delay decomposition approach to stability of linear retarded and neutral systems. Automatica 45(2), 517-524 (2009) 
8. Zhu, Y., Liu, M.: Permanence and extinction in a stochastic service-resource mutualism model. Appl. Math. Lett. 69, 1-7 (2017)

9. Liu, M., Fan, M.: Permanence of stochastic Lotka-Volterra systems. J. Nonlinear Sci. Appl. 27(2), 425-452 (2017)

10. Liu, M., Wang, K., Wu, Q.: Survival analysis of stochastic competitive models in a polluted environment and stochastic competitive exclusion principle. Bull. Math. Biol. 73(9), 1969-2012 (2011)

11. Li, X., Gray, A., Jiang, D., Mao, X.: Sufficient and necessary conditions of stochastic permanence and extinction for stochastic logistic populations under regime switching. J. Math. Anal. Appl. 376(1), 11-28 (2011)

12. Jiang, D., Shi, N., Li, X.: Global stability and stochastic permanence of a nonautonomous logistic equation with random perturbation. J. Math. Anal. Appl. 340(1), 588-597 (2008)

13. Zhao, J., Zhang, Z., Ju, J.: Necessary and sufficient conditions for permanence and extinction in a three dimensional competitive Lotka-Volterra system. Appl. Math. Comput. 230, 587-596 (2014)

14. Chen, F., Chen, X., Huang, S.: Extinction of a two species non-autonomous competitive system with Beddington-DeAngelis functional response and the effect of toxic substances. Open Math. 14(1), 1157-1173 (2016)

15. Chen, F., Ma, Z., Zhang, H.: Global asymptotical stability of the positive equilibrium of the Lotka-Volterra prey-predator model incorporating a constant number of prey refuges. Nonlinear Anal., Real World Appl. 13(6), 2790-2793 (2012)

16. Hou, J., Teng, Z., Gao, S.: Permanence and global stability for nonautonomous N-species Lotka-Volterra competitive system with impulses. Nonlinear Anal., Real World Appl. 11(3), 1882-1896 (2010)

17. Oca, F.M.D., Pérez, L.: Extinction in nonautonomous competitive Lotka-Volterra systems with infinite delay. Nonlinear Anal. 75(2), 758-768 (2012)

18. Qiu, H., Deng, W:: Optimal harvesting of a stochastic delay competitive Lotka-Volterra model with Levy jumps. Appl. Math. Comput. 317(12), 210-222 (2018)

19. Muroya, Y:: Global stability of a delayed nonlinear Lotka-Volterra system with feedback controls and patch structure. Appl. Math. Comput. 239(239), 60-73 (2014)

20. Huo, H.-F., Li, W.-T.: Positive periodic solutions of a class of delay differential system with feedback control. Appl. Math. Comput. 148(1), 35-46 (2004)

21. Zhen, J., Ma, Z:: Stability for a competitive Lotka-Volterra system with delays. Nonlinear Anal. 51(7), 1131-1142 (2002)

22. Park, J.H.: Stability for a competitive Lotka-Volterra system with delays, LMI optimization approach. Appl. Math. Lett. 18(6), 689-694 (2005)

23. Qiu, J., Cao, J.: Exponential stability of a competitive Lotka-Volterra system with delays. Appl. Math. Comput. 201(1), 819-829 (2008)

24. Sun, Y.G., Meng, F.W.: LMI approach to stability for a competitive Lotka-Volterra system with time-varying delays. Appl. Math. Comput. 194(2), 291-297 (2007)

25. Zhang, L., He, L., Song, Y.: New results on stability analysis of delayed systems derived from extended Wirtinger's integral inequality. Neurocomputing 283, 98-106 (2018)

26. Seuret, A., Gouaisbaut, F.: Wirtinger-based integral inequality: application to time-delay systems. Automatica 49(9), 2860-2866 (2013)

27. Zeng, H.-B., He, Y., Wu, M., She, J.: Free-matrix-based integral inequality for stability analysis of systems with time-varying delay. IEEE Trans. Autom. Control 60(10), 2768-2772 (2015)

28. Zhang, C.-K., He, Y., Jiang, L., Wu, M., Wang, Q.-G.: An extended reciprocally convex matrix inequality for stability analysis of systems with time-varying delay. Automatica 85, 481-485 (2017)

29. Qian, W., Wang, L., Chen, M.Z.Q.: Local consensus of nonlinear multiagent systems with varying delay coupling. IEEE Trans. Syst. Man Cybern. Syst. 48(12), 2462-2469 (2018)

30. Qian, W., Gao, Y., Yang, Y.: Global consensus of multiagent systems with internal delays and communication delays. IEEE Trans. Syst. Man Cybern. Syst. (2018). https://doi.org/10.1109/TSMC.2018.2883108

31. Chen, Y., Wang, Z., Shen, B., Dong, H.: Exponential synchronization for delayed dynamical networks via intermittent control: dealing with actuator saturations. IEEE Trans. Neural Netw. Learn. Syst. 30(4), 1000-1013 (2018)

32. Chen, Y., Wang, Z., Fei, S., Han, Q.-L.: Regional stabilization for discrete time-delay systems with actuator saturations via a delay-dependent polytopic approach. IEEE Trans. Autom. Control 64(3), 1257-1264 (2019)

33. Chen, Y., Fei, S., Li, Y.: Robust stabilization for uncertain saturated time-delay systems: a distributed-delay-dependent polytopic approach. IEEE Trans. Autom. Control 62(7), 3455-3460 (2017)

\section{Submit your manuscript to a SpringerOpen ${ }^{\circ}$ journal and benefit from:}

- Convenient online submission

- Rigorous peer review

- Open access: articles freely available online

- High visibility within the field

- Retaining the copyright to your article

Submit your next manuscript at $>$ springeropen.com 